\title{
AN INNOVATIVE FOOT MODULE WITH EASILY ACCESSIBLE FRONTAL PLANE ADAPTATION ENHANCES THE LOCOMOTION ON UNEVEN GROUND
}

\author{
Björn Altenburg*, Michael Ernst, Thomas Schmalz \\ OttoBock HealthCare GmbH, Germany. \\ *Email: Bjoern.Altenburg@ottobock.com
}

DOI: https://doi.org/10.33137/cpoj.v1i2.32029

\section{INTRODUCTION}

Real-life outdoor walking of amputees is challenged by uneven ground. Uneven ground requires either a component adaptation in the sagittal plane or in frontal plane or both. The lack of adaptability of prosthetic components requires compensational movement strategies by the user. Common energy storing and returning (ESR) feet have some basic flexibility through the carbon structure allowing for some limited adaptation in both planes. For the frontal plane the split toe feature adds some functionality. However, even with split toe the ROM is clearly limited and needs high force impact for minor adaptations. Now there is a novel foot module allowing for $10^{\circ}$ inversion/eversion through a dedicated joint. This study investigates the hypothesis that such a foot module with easily accessible frontal plane adaptation enhances the locomotion on uneven ground.

\section{METHODS}

12 unilateral TT amputees with activity level K3 or higher participated in this study. In addition the data of 10 able-bodied was captured. For the measurements a stationary gait analysis system with 12 Vicon Bonita cameras and 2 Kistler force plates was used. A dedicated marker set was applied that enables the observer to distinguish frontal plane adaptations of the foot between joint adaptation, carbon base deformation and shoe/footshell deformation. Observed situations: walking on level ground, walking on a $5^{\circ}$ cross slope, walking on a $10^{\circ}$ cross slope (all at self-selected walking speed, ), standing on level ground, standing on a $5^{\circ}$ cross slope, standing on a $10^{\circ}$ cross slope. All cross slopes were connected to the Kistler force plates. The participants completed the measurement tracks 3 times with 3 setups: their everyday foot (individual ESR), the novel foot module, a reference ESR (Triton LP, Ottobock or Proflex LP, Össur). The accommodation time to the different feet was minimum 4 weeks each. The order of setups and type of reference
ESR was randomized. Next to the biomechanical measurements the participants walked an indoor course (gravel, obstacles, cross slopes, tight turns) and completed questionnaires with respect to socket comfort, safety and overall satisfaction during the indoor course and their $>4$ weeks daily use of the components.

\section{RESULTS}

For the carbon structure of the novel foot module an early and nearly full adaptation to the cross slope at the beginning of mid stance was measured. The reference ESR feet showed significant $(\mathrm{p}<0,05)$ less adaptation with maxima at the end of terminal stance. The COP path of the novel foot clearly shows a more physiological pattern (referring to measurements of the sound side and control group) compared to the ESR feet. The measured external knee adduction moment (EAM) at the $10^{\circ}$ valley condition was significantly reduced with the novel foot module. The questionnaires report significantly higher ratings for perceived safety and socket comfort using the novel foot module compared to the reference ESR foot used in the study. Eight out of twelve TT users preferred the novel foot module over the reference ESRs for their daily routine.

\section{CONCLUSION}

The study results confirm benefits of the novel foot module when ambulating on cross slopes as one condition of uneven ground. Especially for TT amputees it is crucial to minimize recurring improper frontal knee loads on the affected side. Such component functionality contributes to minimize frontal knee loads. Due to the faster and more comprehensive adaptation to the cross slope condition, less compensation is required and users feel more safe and comfortable when ambulating on uneven ground using the novel foot module.

\section{SIGNIFICANCE}

Few authors have investigated lower limb amputee gait on cross slopes ${ }^{1,2,3,4,5}$ with main focus on kinematics and compensational strategies during swing phase. This 
study focuses on stance phase. In addition it's the first study comparing different foot components on cross slopes and thus makes a valuable contribution to the literature of lower limb amputee's gait.

\section{REFERENCES}

1. Starholm IM et al. Energy expenditure of transfemoral amputees walking on a horizontal and tilted treadmill simulating different outdoor walking conditions. Prosthet Orthot Int; 2010; 34; 184-94. DOI: $\underline{10.3109 / 03093640903585016}$

2. Hak I et al. Walking in an unstable environment: Strategies used by transtibial amputees to prevent falling during gait. Arch Phys Med Rehabil; 2013; 94; 2186-93. DOI: $\underline{\text { 10.1016/j.apmr.2013.07.020 }}$

3. Villa $\mathrm{C}$ et al. Evolution of vaulting strategy during locomotion of individuals with transfemoral amputation on slopes and cross-slopes compared to level walking. Clin Biomech; 2015; 30; 623-8. DOI: 10.1016/j.clinbiomech.2015.03.022

4. Sinitski EH et al. Fixed and self-paced treadmill walking for able-bodied and transtibial amputees in a multi-terrain virtual environment. Gait Posture; 2015; 41; 568-73. DOI: 10.1016/j.gaitpost.2014.12.016

5. Villa C et al. Cross-Slope and Level Walking Strategies During Swing in Individuals With Lower Limb Amputation. Arch Phys Med Rehabil; 2017; 98; 1149-57. DOI: 10.1016/j.apmr.2016.10.007

\section{DISCLOSURE}

Authors are full time employees of the Ottobock SE \& Co. $\mathrm{KGaA}$. 